\title{
Nucleation of membrane adhesions
}

\author{
Cheng-Zhong Zhang * and Zhen-Gang Wang ${ }^{\dagger}$ \\ Division of Chemistry and Chemical Engineering, California Institute of Technology, Pasadena, California 91125, USA
}

(Received 15 September 2007; revised manuscript received 11 December 2007; published 11 February 2008)

Recent experimental and theoretical studies of biomimetic membrane adhesions [Bruinsma et al., Phys. Rev. E 61, 4253 (2000); Boulbitch et al., Biophys. J. 81, 2743 (2001)] suggested that adhesion mediated by receptor interactions is due to the interplay between membrane undulations and a double-well adhesion potential, and should be a first-order transition. We study the nucleation of membrane adhesion by finding the minimum-energy path on the free energy surface constructed from the bending free energy of the membrane and the double-well adhesion potential. We find a nucleation free energy barrier around $20 k_{\mathrm{B}} T$ for adhesion of flexible membranes, which corresponds to fast nucleation kinetics with a time scale of the order of seconds. For cell membranes with a larger bending rigidity due to the actin network, the nucleation barrier is higher and may require active processes such as the reorganization of the cortex network to overcome this barrier. Our scaling analysis suggests that the geometry of the membrane shapes of the adhesion contact is controlled by the adhesion length that is determined by the membrane rigidity, the barrier height, and the length scale of the double-well potential, while the energetics of adhesion is determined by the depths of the adhesion potential. These results are verified by numerical calculations.

DOI: 10.1103/PhysRevE.77.021906

PACS number(s): 87.17.-d, 87.16.D-, 82.60.Nh

\section{INTRODUCTION}

Cell adhesion is crucial to many biological processes, including cell differentiation and division, signal transduction, and immunological responses [1-3]. Many different interactions are involved in adhesions in vivo: lock-and-key type interactions between proteins [4], force-induced signaling, reorganization of the actin filaments and cortex [5], and various generic physical forces [6]. Despite the complexity of these interactions, many features of cell adhesion can be qualitatively understood from basic physical principles [7-18].

In contrast to adhesion mediated by generic interactions such as the van der Waals or electrostatic forces, biological adhesions are induced by specific binding between proteins with complementary domains - receptors and ligands. Other interactions provide different regulation mechanisms that fortify (e.g., cytoskeleton reorganization) or destabilize (e.g., repeller molecules) the adhesion contact. While adhesion receptors play the major role and are extensively studied, deadhesion forces are crucial to ensure specificity of the desired adhesion $[14,16]$. The interplay between attractive specific and (usually repulsive) nonspecific forces is a recurring theme in cell adhesion and provides delicate control over the adhesion-deadhesion kinetics in dynamic processes such as cell migration or leukocyte rolling [19].

While receptors and their ligands have been the focus of biological studies over the past decades, the physical carrier of these proteins - the cell membrane-has been extensively studied by physicists and biophysicists since the fluid-mosaic model was proposed by Singer and Nicolson [20]. Membranes are composed of self-assembled lipid molecules and

\footnotetext{
*Current address: Immune Disease Institute, Harvard Medical School, Boston, MA 02115.

†Corresponding author.zgw@cheme.caltech.edu
}

form vesicles in aqueous solutions of typical sizes up to $10 \mu \mathrm{m}$. The physics of fluid or solid membranes are well studied and comprehensively summarized in Refs. [6,21,22]. In particular the interactions between flexible membranes have been studied by Lipowsky $[5,23]$ and Leibler and coworkers [24,25].

Recent advances in bioengineering techniques have enabled studies of adhesion of biomimetic membranes mediated by specific and nonspecific interactions. Sackmann and co-workers [26,27] have designed self-assembled vesicles and monolayers supported by a polymer cushion to mimic cell membranes and the extracellular matrix; in the membranes they incorporated specific proteins (to mimic ligandreceptor binding) and glycolipids (to mimic the glycocalyx), as well as other additives to stabilize the vesicle. This system provides the first artificial system incorporating key elements of cell adhesion and allows systematic studies of the mechanics and dynamics of adhesion without complications due to factors present in biological cells.

Based on in vitro experiments using biomimetic vesicles, Sackmann, Bruinsma, and coworkers [14,16,18] found that cell adhesion is controlled by a double-well potential: a weak-adhesion state at a large surface separation due to generic van der Waals interactions between the lipids and a strong-adhesion state at a small surface separation due to ligand-receptor binding; membrane undulation and glycolipids induce repulsive forces that constitute the barrier between the two minima. The adhesion proceeds in three steps $[15,17,28,29]$. First, small adhesion contacts are formed which are most likely induced by membrane undulations; such a process is an activated process with a nucleation barrier larger than $10 k_{\mathrm{B}} T$. Following nucleation, receptors diffuse into the adhesion contacts and the contact area grows accompanied by a depletion of repellers (glycolipids); this is the growth step. Finally, after receptors are depleted, the adhesion contacts evolve like coarsening in a phase separation: the number of adhesion contacts decreases and various small focal contacts are formed with high densities of receptors, 
accompanied by a possible decrease in the total area of contact. The entire process is likened to the wetting transition [16], and phenomenological parameters derived from the wetting process, such as the surface tension, the spreading pressure, and the contact angle, can be measured and related to the underlying parameters of membrane adhesion, including the elastic moduli of the membrane and the receptor binding affinity $[14,15,30]$.

The conformations of adhered membranes are recorded in situ by reflection interference contrast microscopy (RICM) [31,32], which provides direct observation and measurements of the formation and growth of adhesion plaques. However, RICM is unable to resolve adhesion contacts smaller than $300 \mathrm{~nm}$ [15] and therefore cannot give direct information on the nucleation process. On the other hand, scaling arguments and Monte Carlo studies [23,33] usually provide only order-of-magnitude estimates and do not yield quantitative results that are experimentally testable. In particular, the nucleation step of the adhesion process has never been studied.

In this paper we present a systematic study of the nucleation step of adhesion. Following Refs. [14,16,17], we assume the "minimal" model of membrane adhesion that consists of the elastic deformation energy of the flexible membrane and a double-well adhesion potential: this minimal model preserves the key features of cell adhesion and also appears in systems with ligand-receptor interactions at different length ranges-e.g., the immunological synapse [34]. From a scaling analysis we find that the membrane shapes are governed by the adhesion length $R_{0}$ which is determined from the bending rigidity of the membrane $\kappa$ and the adhesion potential; the energy barrier is controlled by the energy scale $F_{0}=\sqrt{\kappa V_{\text {eff }} L_{0}^{2}}$, where $V_{\text {eff }}$ is the effective potential barrier height and $L_{0}$ is the characteristic length of the adhesion potential.

For typical values of the parameters in vesicle adhesion, the energy scale $F_{0} \gg k_{\mathrm{B}} T$; therefore, adhesion is a first-order transition and nucleation proceeds along the "minimumenergy path" governed by the effective potential (free energy). Using the string method by E et al. [35], we calculate the minimum-energy path from the weakly bound state to a well-developed adhesion contact. We find that the typical energy barrier for adhesion between flexible membranes is about $(20-30) k_{\mathrm{B}} T$, corresponding to a time scale of $0.1-1000 \mathrm{~s}$. For adhesion of cells with actin cortices, which have much larger bending moduli, the nucleation barrier is higher and could be insurmountable by thermal undulations. Active processes, such as actin reorganization and cell signaling, are therefore probably important for providing additional mechanisms for stabilizing the adhesion contact. In particular, we point out that in the binding between a T-cell and an antigen-presenting cell, the energy barrier of T-cell receptor binding controls the final morphology.

\section{MODEL AND SOLUTION}

\section{A. Model description}

The thickness of a self-assembled monolayer or bilayer is about $10-50 \mathrm{~nm}$, usually negligible compared to its spatial

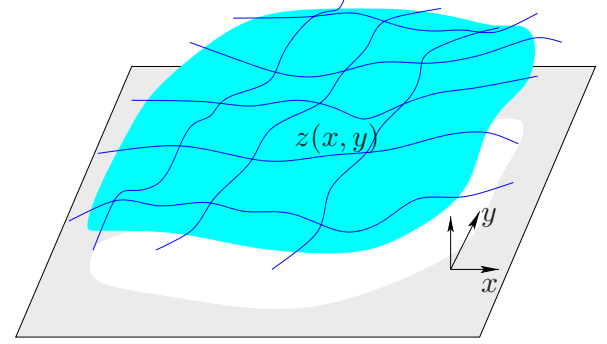

FIG. 1. (Color online) Monge representation of a near-flat membrane shape.

extension $(\sim 10 \mu \mathrm{m})$. Therefore the macroscopic behavior of membranes is mostly determined by their geometric shapes and to a good approximation independent of the microscopic degrees of freedom of the consistituent amphiphilic molecules. Flexible membranes as random surfaces have been extensively studied in the past decades by physicists; theoretical models and results are summarized in Ref. [6]. For cell membranes or self-assembled monolayers with biological relevance, see Refs. [5,22]; a more up-to-date review of simulation methods and phenomenological models can be found in Ref. [36].

For a single membrane that is homogeneous, smooth, and noninteracting, Helfrich [37] proposed that up to secondorder derivatives with respect to the local coordinates of the membrane shape, the elastic energy of a deformed membrane is given by

$$
H_{\mathrm{e}}=\int_{S}\left[\sigma+\frac{1}{2} \kappa\left(H-H_{0}\right)^{2}+\bar{\kappa} K\right] d A .
$$

Here $\sigma$ is the (local) surface tension conjugate to the surface area; $\kappa$ and $\bar{\kappa}$ are elastic moduli known as the bending rigidity and the Gaussian rigidity coupled to the mean curvature $H$ and the Gaussian curvature $K$, respectively; $H_{0}$ is the spontaneous curvature, which is zero for a symmetric bilayer and assumed to be the case here. The integral is over the entire membrane area.

In this paper we study the adhesion between a flexible membrane and a rigid flat surface, corresponding to the experimental system studied by Bruinsma et al. [14]. In this setting the membrane is almost flat and does not intersect itself; hence, the separation between the membrane and the flat surface provides a natural representation of the membrane shape (cf. Fig. 1), $z=z(x, y)$, also called the Monge representation. ${ }^{1}$ The elastic energy of the deformed membrane up to the second order derivatives of $z(x, y)$ is given by

\footnotetext{
${ }^{1}$ In the case of adhesion between two membranes, the elastic energy is divided into two parts: one due to deformations of the "center of mass" of the binary system, the other dependent on the relative separation between the membranes; after integrating out the center-of-mass deformations, one can write the elastic energy dependent on the relative separation in the same form as above with the additive bending rigidity (cf. Ref. [13]):

$$
\kappa^{-1}=\kappa_{1}^{-1}+\kappa_{2}^{-1} \text {. }
$$
}




$$
H_{\mathrm{e}}=\int\left\{\frac{\kappa}{2}[\Delta z(x, y)]^{2}+\sigma[\nabla z(x, y)]^{2}\right\} d x d y .
$$

The elastic energy gives the "kinetic" part of the Hamiltonian.

Next we consider the interaction potential between the adhesion surfaces. Generic (nonspecific) interactions, including the van der Waals interaction, electrostatic interaction, and hydration forces (see, for example, Ref. [6], Chap. 3) result in a potential $V_{\mathrm{g}}$ with a minimum around $10-100 \mathrm{~nm}$ $[14,28,38]$.

The interactions mediated by receptors and repellers on the membrane have been calculated using a molecular model in our previous paper. ${ }^{2}$ The interaction potential $V_{\mathrm{s}}\left(z ; \phi_{\mathrm{i}}\right)$ depends on the local separation between surfaces $z(\mathbf{r})$ and the receptor densities $\phi_{\mathrm{i}}(\mathbf{r})$. For homogeneously distributed immobile receptors on the substrate (on the time and length scales of interest), $V_{\mathrm{s}}$ is a function of the surface separation $z$ only. When the receptors are mobile, their distribution $\phi_{\mathrm{i}}(\mathbf{r})$ will be coupled to the local separation $z(\mathbf{r})$. Here we assume that nucleation involves a considerable barrier and is therefore slow; this corresponds to, e.g., vesicle adhesion with a high repeller concentration [40]. In this regime, states along the nucleation path will be close to equilibrium and we may use a quasi-equilibrium free energy in terms of any convenient coarse-grained variable, with other variables eliminated by minimizing the free energy over them at a fixed value of the chosen variable. For our current problem, this means that the molecular distributions are averaged to give an effective interaction potential $V(z)$ that only depends on the separation. Provided the quasi-equilibrium assumption holds, the actual nucleation path in the two-order-parameter space $z(\mathbf{r})$ and $\phi_{\mathrm{i}}(\mathbf{r})$ should be close to the minimum-energy path determined by using only $V(z)$. In particular, the saddle point on the free energy landscape, where the free energy gradient vanishes, should be completely identical, whether the free energy surface is given using the two-order-parameter representation in terms of both $z(\mathbf{r})$ and $\phi_{\mathrm{i}}(\mathbf{r})$ or using a reduced representation in terms of $z(\mathbf{r})$ only.

The total Hamiltonian is given by $[\mathbf{r}=(x, y)]$

$$
H[z(\mathbf{r})]=\int\left\{\frac{\kappa}{2}[\Delta z(\mathbf{r})]^{2}+\sigma[\nabla z(\mathbf{r})]^{2}+V[z(\mathbf{r})]\right\} d^{2} \mathbf{r} .
$$

$V(z)$ is the superposition of the generic potential $V_{\mathrm{g}}(z)$ and the specific binding interaction potential $V_{\mathrm{s}}(z)$.

The free energy (effective potential) of the model given by Eq. (3) can be calculated using the standard field-theoretic method by integrating out short-wavelength fluctuations of the separation variable $z(\mathbf{r})[34,41]$. In particular, membrane undulations induce an effective repulsion which contributes to the overall interaction potential $V[z(\mathbf{r})][5]$. Since these fluctuation effects are not our main focus here, we apply a mean-field approximation and assume the free energy takes the same form as the Hamiltonian with renormalized elastic

\footnotetext{
${ }^{2}$ See Ref. [50] and also Refs. [12,14,39] for phenomenological treatments.
}

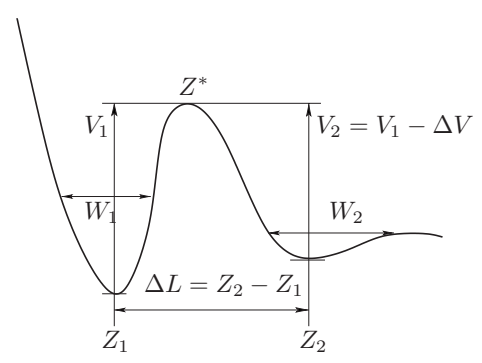

FIG. 2. Schematic representation of the interaction potential $V(z)$. The shape of the potential is similar to that calculated from a phenomenological model in Ref. [14]. Only the double-well part of the potential is relevant for our calculations; the potential is characterized by the barrier position $z^{*}$ and the positions $Z_{1}, Z_{2}$ and the depths $W_{1}, W_{2}$ of the minima. In our numerical calculations we choose the functional form for $V(z)$ such that the widths $W_{1} \approx Z_{1}$ and $W_{2} \approx Z_{2}$ for fast convergence in numerical calculations.

constants and interaction potential; these renormalized parameters are experimentally measurable.

With these approximations we can write the effective potential of our model as

$$
F[z(\mathbf{r})]=\int\left\{\frac{\kappa}{2}[\Delta z(\mathbf{r})]^{2}+\sigma[\nabla z(\mathbf{r})]^{2}+V[z(\mathbf{r})]\right\} d^{2} \mathbf{r} .
$$

The adhesion (interaction) potential $V(z)$ has a double-well shape $[14,16]$ and is characterized by the depths and locations of the minima, as is schematically shown in Fig. 2. The parameters in Eq. (4) have been measured by Sackmann and co-workers in several systems (see Ref. [11], p. 195, and Refs. $[16,18,29,30,42]) . \kappa$ is about $20 k_{\mathrm{B}} T$ for a bilayer membrane and of order $500 k_{\mathrm{B}} T$ for the actin cortices. $\sigma$ is related to the so-called capillary length [18]

$$
R_{\mathrm{c}}=\sqrt{\kappa / \sigma}
$$

which defines the length scale above which surface tension becomes important. Typical values for $R_{\mathrm{c}}$ are about $0.1-1 \mu \mathrm{m}[14,18]$. Generally the size of adhesion plaques in the initial stage of adhesion is smaller than $R_{\mathrm{c}}$; therefore, the surface energy is insignificant compared with the bending energy.

For clarity of our discussion it is convenient to scale the separation $z(\mathbf{r})$ and the radial coordinate $r$ by the natural length scales arising from the adhesion potential and the membrane elasticity. Introducing a lateral length scale $R_{0}$, a vertical length scale $L_{0}$, and an interaction energy scale $V_{2}$ (note that $V_{2}$ is given in units of $k_{\mathrm{B}} T$ per unit area) and performing the rescaling

$$
r / R_{0} \rightarrow r, \quad l / L_{0} \rightarrow l, \quad z / L_{0} \rightarrow z, \quad V / V_{2} \rightarrow v,
$$

we obtain

$$
F[z]=\int\left[\frac{\kappa L_{0}^{2}}{2 R_{0}^{2}}\left(\nabla^{2} z\right)^{2}+\gamma L_{0}^{2}(\nabla z)^{2}+V_{2} R_{0}^{2} v(z)\right] d^{2} \mathbf{r} .
$$

In the rigidity-dominant regime, we choose 


$$
V_{2} R_{0}^{2}=\frac{\kappa L_{0}^{2}}{R_{0}^{2}}
$$

such that the length scales are determined by the adhesion potential $V(z)$ and the bending rigidity. This leads to

$$
F[z]=\sqrt{\kappa V_{2} L_{0}^{2}} \int\left[\frac{1}{2}\left(\nabla^{2} z\right)^{2}+\frac{\Sigma}{2}(\nabla z)^{2}+v(z)\right] d^{2} \mathbf{r},
$$

where the rescaled surface tension is

$$
\Sigma=\frac{2 \sigma L_{0}}{\sqrt{\kappa V_{2}}}=\frac{2 R_{0}^{2}}{R_{\mathrm{c}}^{2}}
$$

and

$$
R_{0}=\left(\frac{\kappa L_{0}^{2}}{V_{2}}\right)^{1 / 4}
$$

is the adhesion length (similar to the "persistence length" defined in Ref. [18]), which controls the boundary width of the adhesion contact. In general $R_{0} \sim 10 \mathrm{~nm} \ll R_{\mathrm{c}}$; therefore, the surface tension term is unimportant.

The combination $\sqrt{\kappa V_{2} L_{0}^{2}}$ (the lateral length scale $L_{0}$ is defined later) sets the magnitude of the free energy. If $\kappa V_{2} L_{0}^{2} \gg 1$, then the minima of $V(z)$ are separated by a large barrier (cf. Refs. [5,23]). In this regime, thermal fluctuations do not affect the physics at length scale $L_{0}$, and we can apply the mean-field capillary approximation.

\section{B. Scaling analysis of the nucleation barrier}

Lipowsky and co-workers have systematically studied the dynamics and thermodynamics of membrane adhesion mediated by a double-well potential by scaling arguments and Monte Carlo simulations [40,43]. They distinguish between two types of transitions: the unbound-bound transition-i.e., transition from unbound membranes infinite apart to bound within the double-well potential - and the lateral phase separation, corresponding to transition between membrane states at different separations of the two potential minima. The unbound-bound transition is determined by the potential depths and widths, while the lateral phase separation is controlled by the barrier height and the separation between the minima. In this paper we focus on the transition from the loosely bound metastable minimum to the global minimum of the potential, which is related to the lateral phase separation. Experimental studies found that the biomimetic vesicle adhesion is a first-order transition with a sizable barrier $[15,28]$; therefore, it should proceed via a nucleation-andgrowth pathway. Our study is geared primarily at the vesicle adhesion problem, but we believe that some of our results are also applicable to cell adhesion.

In this subsection we perform a scaling analysis of the nucleation dynamics using the classical capillary approximation. We assume that initially the membrane is in a loosely bound state at a larger separation $Z_{2}$ in a flat shape. Nucleation of an adhesion contact is driven by membrane undulations which result in a "droplet" as shown in Fig. 3. In a mean-field picture, the boundary of the adhesion droplet is

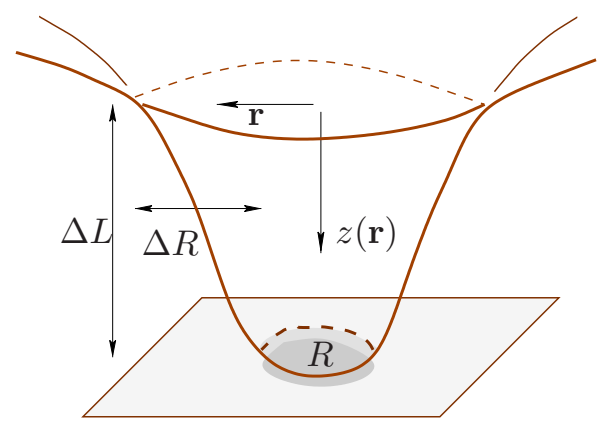

FIG. 3. (Color online) Illustration of a regular adhesion droplet.

regular (a simple curve) and we assume the droplet to be axisymmetric and the membrane deviation $z(r)$ to be a function of the radial coordinate.

If the potential depths are comparable- $V_{1} \approx V_{2}$, $\Delta V \ll V_{2}$ - the length scale $L_{0}$ associated with the adhesion potential $V(z)$ is naturally chosen to be the separation between the minima $\Delta L=Z_{2}-Z_{1}$; otherwise, when $\Delta V \geqslant V_{2}, L_{0}$ should be taken to be the separation of the metastable minimum $\left(V_{2}\right)$ from the barrier $Z_{2}-Z^{*}$.

In the first case $V_{1} \approx V_{2}$, there is a well-defined adhesion "nucleus" which has size $R$ in the interior and an "interfacial ring" of width $\Delta R$ [cf. Fig. 3]. The length scales are

$$
\begin{gathered}
L_{0}=\Delta L, \\
R_{0}=\left(\frac{\kappa \Delta L^{2}}{V_{2}}\right)^{1 / 4},
\end{gathered}
$$

and the energy scale is

$$
F_{0}=\sqrt{\kappa V_{2} \Delta L^{2}} .
$$

From scaling analysis we have

$$
\nabla^{2} z \sim \frac{L_{0}}{\Delta R^{2}} \Rightarrow \kappa \int\left(\nabla^{2} z\right)^{2} d A \sim \frac{\kappa R L_{0}^{2}}{\Delta R^{3}}
$$

the interfacial energy scales as

$$
R \Delta R V_{2} \text {. }
$$

Combining these two contributions we find

$$
\Delta R \sim R_{0}=\left(\frac{\kappa \Delta L^{2}}{V_{2}}\right)^{1 / 4}
$$

and the line tension is

$$
\Gamma \approx \Delta R V_{2} \sim \kappa^{1 / 4} V_{2}^{3 / 4} \Delta L^{1 / 2} .
$$

The total free energy of the droplet is

$$
F=-\pi R^{2} \Delta V+\pi R \Gamma .
$$

At the critical radius $R^{\ddagger}$ the free energy attains a maximum and we have

$$
R^{\ddagger} \sim \frac{V_{2}}{\Delta V} \Delta R=\frac{V_{2}}{\Delta V}\left(\frac{\kappa \Delta L^{2}}{V_{2}}\right)^{1 / 4}=\frac{V_{2}}{\Delta V} R_{0},
$$




$$
F^{\ddagger} \sim \frac{V_{2}^{2}}{\Delta V} \Delta R^{2}=\frac{V_{2}}{\Delta V}\left(\kappa V_{2} \Delta L^{2}\right)^{1 / 2}=\frac{V_{2}}{\Delta V} F_{0} .
$$

When the potential depth difference is larger than the barrier height $-V_{2} / \Delta V \leqq 1$ - the length scale

$$
L_{0} \approx\left(Z_{2}-Z^{*}\right)=L_{2}
$$

and the radius $R^{\ddagger}$ is comparable to the boundary width $\Delta R$. The above results become

$$
\begin{gathered}
R^{\ddagger} \sim \Delta R \sim R_{0}=\left(\frac{\kappa L_{2}^{2}}{V_{2}}\right)^{1 / 4}, \\
F^{\ddagger} \sim \pi R_{0}^{2} V_{2}=F_{0} .
\end{gathered}
$$

From Eqs. (12), (13), $\left(12^{\prime}\right)$, and (13') we see that $R_{0}$ and $F_{0}$ control the length $(R$ and $\Delta R)$ and energy $(F)$ scales, respectively. The dependence of $R_{0}$ on the barrier height $R_{0}$ $\sim V_{2}^{-1 / 4}$ is different from typical correlation length in liquidgas systems, which corresponds to $R_{0} \sim V_{2}^{-1 / 2}$. This difference is due to the appearance of the square Laplacian term as opposed to a square gradient term in the free energy functional.

We note that the capillary analysis is valid only if $F_{0} \gg k_{\mathrm{B}} T$. When $F_{0} \sim k_{\mathrm{B}} T$, membrane undulations result in adhesion contacts with irregular shapes. In particular,

$$
\kappa V_{2} \Delta L^{2} \approx 1
$$

marks the crossover of the binding transition from first order to second order [23].

\section{Minimum-energy-path calculation}

In the mean-field picture, nucleation proceeds along the "minimum-energy path," which corresponds to the "valley" on the free energy landscape. Specifically we want to find the minimum-energy path $z(r, s)(0 \leq s \leq 1)$ that connects the initial state $z(r, s=0)$ (flat membrane) and the terminal state $z(r, s=1)$ (a well-developed adhesion droplet).

The minimum-energy path (MEP) is defined such that the tangent along the path $\nabla_{s} z(r, s)$ is parallel to the free energy gradient $\delta F[z] / \delta z$ at any point on the path, or put in mathematical terms,

$$
\begin{aligned}
\left(\frac{\delta F[z]}{\delta z}\right)^{\perp} & =\frac{\delta F[z]}{\delta z} \cdot(\mathbf{I}-\hat{s} \hat{s})=0, \\
\hat{s} & =\frac{\nabla_{s} z(r, s)}{\left\|\nabla_{s} z(r, s)\right\|} .
\end{aligned}
$$

To calculate $z(r, s)$ we adopt the string method by $\mathrm{E}$ and co-workers [35], which is a modified steepest descent

$$
\frac{\partial z(R, s ; t)}{\partial t}=-\frac{\delta F[z]}{\delta z} \cdot(\mathbf{I}-\hat{s} \hat{s})+\lambda \hat{s} .
$$

Here $\lambda$ is a Lagrange multiplier which is used to fix the parametrization $s$. The choice of $\lambda$ is arbitrary, and we adopt the same parametrization as given in Ref. [35], which re- quires the points be uniformly separated along the path,

$$
\left\|\nabla_{s} z(r, s)\right\|=\text { const. }
$$

This parametrization has a close form expression for $\lambda$.

In our model, the free energy gradient is given by

$$
\frac{\delta F[z]}{\delta z}=\Delta^{2} z-\Sigma \Delta z+v^{\prime}(z)
$$

The initial state $z(r, 0)$ is a flat surface $z(r, 0)=Z_{2}$ (at the metastable minimum), while the terminal state $z(r, 1)$ needs to be a well-developed adhesion droplet that has passed the nucleation barrier. To find the final state, we impose a parabolic adhesion droplet whose frontier has passed the barrier $Z^{*}$ (cf. Fig. 2) and let it evolve along the free energy gradient (steepest descent). If the droplet size is large enough, it will reach steady growth after evolving for some steps; this final profile is taken as the terminal state $z(r, 1)$. The initial path $z(r, s ; t=0)$ is generated by a simple linear interpolation between $z(r, 0)=Z_{2}$ and $z(r, 1)$. Because the terminal state $z(r, 1)$ is not stationary (the droplet will still grow), it will also evolve in the iteration using Eq. (16); therefore, our MEP resembles a "rope" with one end fixed, instead of the "string" with both ends fixed as in the original paper by E $e t$ al.

We adopt an explicit forward time splitting for the potential $V(z)$ and an implicit splitting for the differential operators, which ensures fast convergence. ${ }^{3}$ Iteration stops when the maximum free energy of the reaction path, $\max _{s} F[z(s)]$, reaches a constant and the maximum residual gradient $\max _{s}\left\{\nabla F(z)^{\perp}\right\}$ is used to test the accuracy of convergence. In the next section we discuss results of this minimum-energypath calculation.

\section{RESULTS AND DISCUSSION}

In this section we discuss numerical results of the MEP calculations. Before the discussion we first estimate the typical length and energy scales associated with the adhesion process. The bending rigidity $\kappa$ is about $20 k_{\mathrm{B}} T$ for bilayer membranes and $500 k_{\mathrm{B}} T$ for the actin cortex $[16,18]$. The separation $\Delta L$ is between 5 and $50 \mathrm{~nm}$, depending on the size of the receptors $[14,44]$, and we take it to be $5 \mathrm{~nm}$. The barrier height $V_{2}$ is estimated to be $10^{-5} \mathrm{~J} / \mathrm{m}^{2} \quad(2.4$ $\times 10^{-3} k_{\mathrm{B}} T / \mathrm{nm}^{2}$ at $\left.T=300 \mathrm{~K}\right)$ [14]. Therefore the energy scale for flexible membranes is

$$
F_{0}=\left(\kappa V_{2} \Delta L^{2}\right)^{1 / 2} \approx 1.2 k_{\mathrm{B}} T,
$$

which indeed reflects flexibility of the membrane. The real barrier $F^{*}$ ranges from $5 k_{\mathrm{B}} T$ to $30 k_{\mathrm{B}} T$. The lateral length scale is

$$
R_{0}=\sqrt[4]{\kappa \Delta L^{2} / V_{2}} \approx 20 \mathrm{~nm} .
$$

In cell adhesions, the cell membrane and the embedded adhesion molecules (e.g., integrins) are coupled to the cytoskeleton, giving rise to a larger bending rigidity due to the

\footnotetext{
${ }^{3}$ See, for example, the papers in Ref. [51].
} 


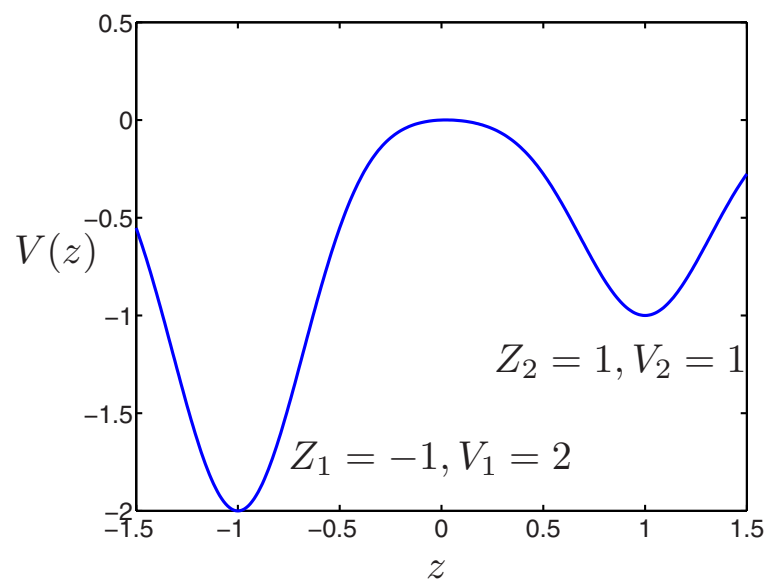

FIG. 4. (Color online) Shape of the potential $V(z)$ for $Z_{1}=-1$, $Z_{2}=1, V_{1}=2$, and $V_{2}=1 ; Z^{*}$ is fixed at the origin.

underlying actin cortex. As the typical separation between anchoring points in an actin network is of order $100 \mathrm{~nm}$, the apparent bending rigidity $\kappa$ will depend on the relevant length scale and lies between $20 k_{\mathrm{B}} T$ (below $100 \mathrm{~nm}$ ) and $500 k_{\mathrm{B}} T$ (around $1 \mu \mathrm{m}$ ). As the actual length scale is unknown a priori and depends on the bending rigidity, here we take as an estimate of $\kappa$ to be between $50 k_{\mathrm{B}} T$ and $100 k_{\mathrm{B}} T$.

The capillary length

$$
R_{\mathrm{c}}=\sqrt{\kappa / \sigma}
$$

is usually of order $0.1 \mu \mathrm{m}$ [18]; hence, the dimensionless surface tension

$$
\Sigma \sim \frac{R_{0}^{2}}{R_{c}^{2}}
$$

is small and we neglect the surface tension term for most of our calculations; we include a brief discussion of its effects after presenting our main results.

We parametrize the scaled potential $V(z)$ as a sum

$$
\begin{aligned}
V(z)= & V_{1}\left[\left(\frac{z}{Z_{1}}-1\right)^{2}-1\right] \exp \left[-4\left(\frac{z}{Z_{1}}-1\right)^{2}\right] \\
& +V_{2}\left[\left(\frac{z}{Z_{2}}-1\right)^{2}-1\right] \exp \left[-4\left(\frac{z}{Z_{2}}-1\right)^{2}\right]
\end{aligned}
$$

which is minimized at $z=Z_{1}$ and $z=Z_{2}$ and has a barrier located at approximately $z=0$. An example is shown in Fig. 4. In most situations the separations of the minima from the barrier $Z^{*}-Z_{1}$ and $Z_{2}-Z^{*}$ are comparable; we choose them to be equal (i.e., the positions of minima are symmetric relative to the barrier position). As the nucleation dynamics depends primarily on a few key features of the double-well potential - the difference between the two energy minima, the barrier height from the metastable minimum, and the spatial distance between the two minima-the specific functional form of the potential is inconsequential. The present one is chosen purely based on convenience.
In Fig. 5 we present two representative nucleation paths. The barrier position $Z^{*}$ is fixed at the origin, and the barrier height is $V_{2}=1$; the potential minima are located at $Z_{1}=-1$ and $Z_{2}=1$, and we choose two depths $V_{1}=1.3$ and $V_{1}=4$, giving potential depth differences $\Delta V=0.3$ and 3 , respectively. Figures 5(a) and 5(c) show the evolution of membrane shapes along the MEP: the membrane conformation evolves in the direction of the arrow; Figs. 5(b) and 5(d) give the free energy along the MEP with (red) circles corresponding to each membrane shape shown on the left. The (red) thick curves in (a) and (c) are the critical shapes corresponding to the maximum free energy along the nucleation contour (saddle point).

In the case that potential depths are comparable, $\Delta V$ $=0.3<V_{2}=1$, we see that the critical droplet is well developed with radius $R^{\ddagger} \approx 7 R_{0}=140 \mathrm{~nm}$ and a boundary width $\Delta R \approx 3 R_{0} \simeq 60 \mathrm{~nm}$ : this is similar to the classical nucleation scenario where capillary approximation applies. For $\Delta V=3$ $>V_{2}$, the critical shape has not formed an adhesion contact yet: the frontier just passes the barrier position $Z^{*}=0$. In this latter case the critical droplet size is about equal to the boundary width $\Delta R$ in the first case.

In the first case $\left(V_{1} \approx V_{2}\right)$, the energy barrier is $51 k_{\mathrm{B}} T$, and in the second case $\left(V_{1} \gg V_{2}\right)$, the energy barrier is about $18 k_{\mathrm{B}} T$. To have a better understanding of the nucleation dynamics, we estimate the characteristic time scales of membrane undulations. By a dimensional analysis, we have

$$
\tau_{\text {un }} \sim \eta L^{3} / k_{\mathrm{B}} T=0.24 \mathrm{~ns}
$$

for $L=1 \mathrm{~nm}$ and viscosity of water $\eta=0.001 \mathrm{~Pa}$. For an energy barrier of $18 k_{\mathrm{B}} T$, the nucleation time is

$$
\tau_{0} \sim \tau_{\mathrm{un}} e^{-F^{\grave{\dot{\hbar}}} / k_{\mathrm{B}} T} \sim 15 \mathrm{~ms},
$$

which suggests fast nucleation dynamics. An energy barrier of $51 k_{\mathrm{B}} T$ is essentially insurmountable. In most biological processes or biomimetic experiments, the nucleation time scale is of order seconds to minutes, which corresponds to an energy barrier of $(20-30) k_{\mathrm{B}} T$. Our results suggest that adhesion driven by membrane undulations is dynamically forbidden if the double-well potential has comparable potential minima. In real processes this is overcome by different mechanisms: in cell adhesion, receptors aggregate locally to high densities to enhance the adhesion strength [14]; furthermore, reorganization of the actin cortex can fortify the adhesion contact; other mechanisms such as dimerization of integrin receptors can also be triggered by cell signaling, which provides a mechanism to stabilize the adhesion contact.

Komura and Andelman [45] studied the membrane shape near the phase boundary under lateral phase separation induced by adhesion and found that the membrane deformation is nonmonotonic near the phase boundary between coexisting phases. Our results show that this nonmonotonic feature is present throughout the adhesion process. As we shall see at the end of this section, this feature is due to the bending energy term; increasing surface tension will diminish this feature. 

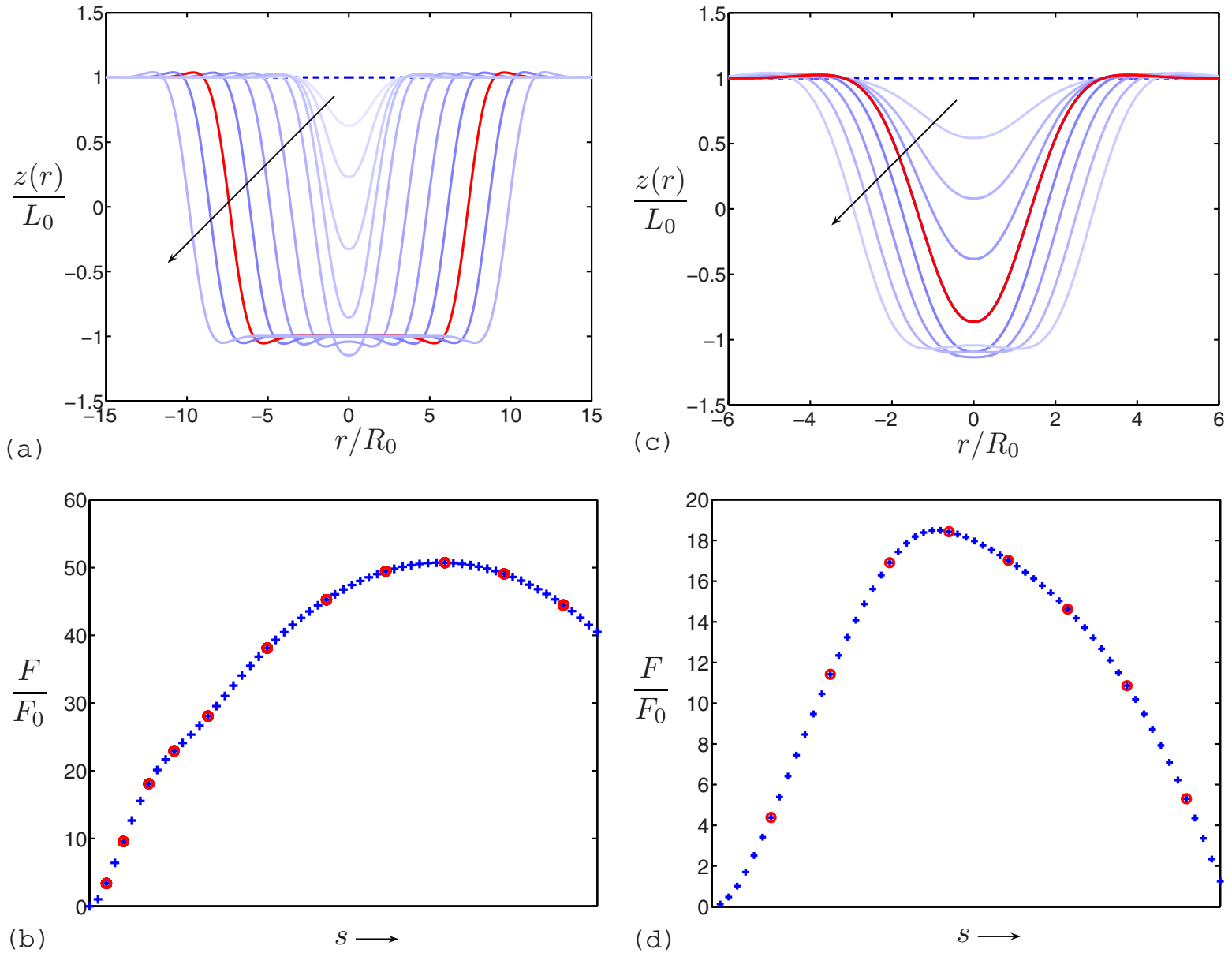

FIG. 5. (Color online) Evolution of the membrane shape [(a) and (c)] and the free energy [(b) and (d)] along the minimum-energy path $(0 \leq s \leq 1$ is the reaction coordinate) for different adhesion potentials. Free energies of the representative shapes in (a) and (c) are shown as (red) circles in (b) and (d), with the most saturated (red) curve giving the critical shape corresponding to the maximum free energy along the contour. (a) and (b) are for the case when the potential depths are comparable, $V_{1}=1$ and $V_{2}=1.3$; (c) and (d) are for $V_{1}=1$ and $V_{2}=4$. In both cases the separation of the potential minima from the potential barrier $Z^{*}$ is 1 , and $\Delta L=2$.

To study the crossover between the two scenarios shown in Fig. 5, we plot the critical membrane shapes for different $\Delta V$ in Fig. 6. We note that for $2 \leq \Delta V \leq 4$ the critical shape is almost invariant: in this regime the barrier height $V_{2}$ is small compared to the potential depth difference $\Delta V$ and nucleation is determined by the potential near the metastable minimum at $Z_{2}$ and independent of $\Delta V$. On the other hand, when $\Delta V$ is small, the critical shape has a well-developed adhesion contact with increasing radii as $\Delta V$ becomes smaller, and one can compare the critical radius and free energy with scaling results from the capillary approximation.

In Fig. 7 we plot the free energy barrier $F^{\ddagger}$ (maximum on the MEP) and the critical nucleus $R^{\ddagger}$, defined as the radius of the critical profile within the adhesion minimum [with $z(r)$ $<0]$. The plot is on a log-log scale. Scaling arguments imply that when $\Delta V \ll V_{2}$, the free energy barrier and the critical nucleus both scale as $1 / \Delta V$. Numerical results indeed confirm this scaling. When $\Delta V \gg V_{2}$, scaling arguments suggest that $\Delta V$ is irrelevant; this trend also holds approximately.

Inspecting Fig. 6 we notice that the boundary shapes of the critical profiles at different $\Delta V$ are similar, which implies that they are controlled by the same length scale. Scaling

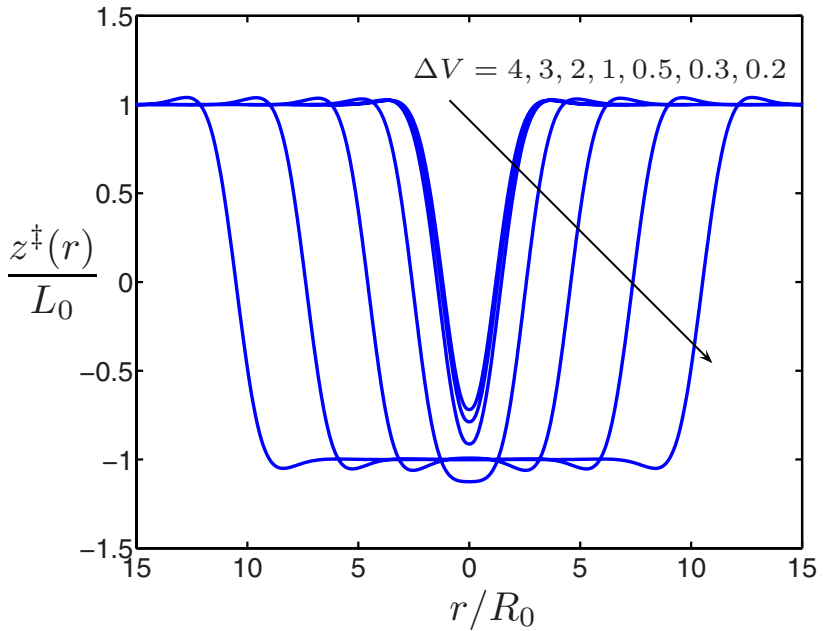

FIG. 6. (Color online) Critical membrane shapes at different potential depths. The positions of the minima are the same as in Fig. 5, $Z_{1}=-1$ and $Z_{2}=1 . \quad V_{2}=1$ and $V_{1}=V_{2}+\Delta V$ with $\Delta V$ $=4,3,2,1,0.5,0.3,0.2$ along the arrow. 

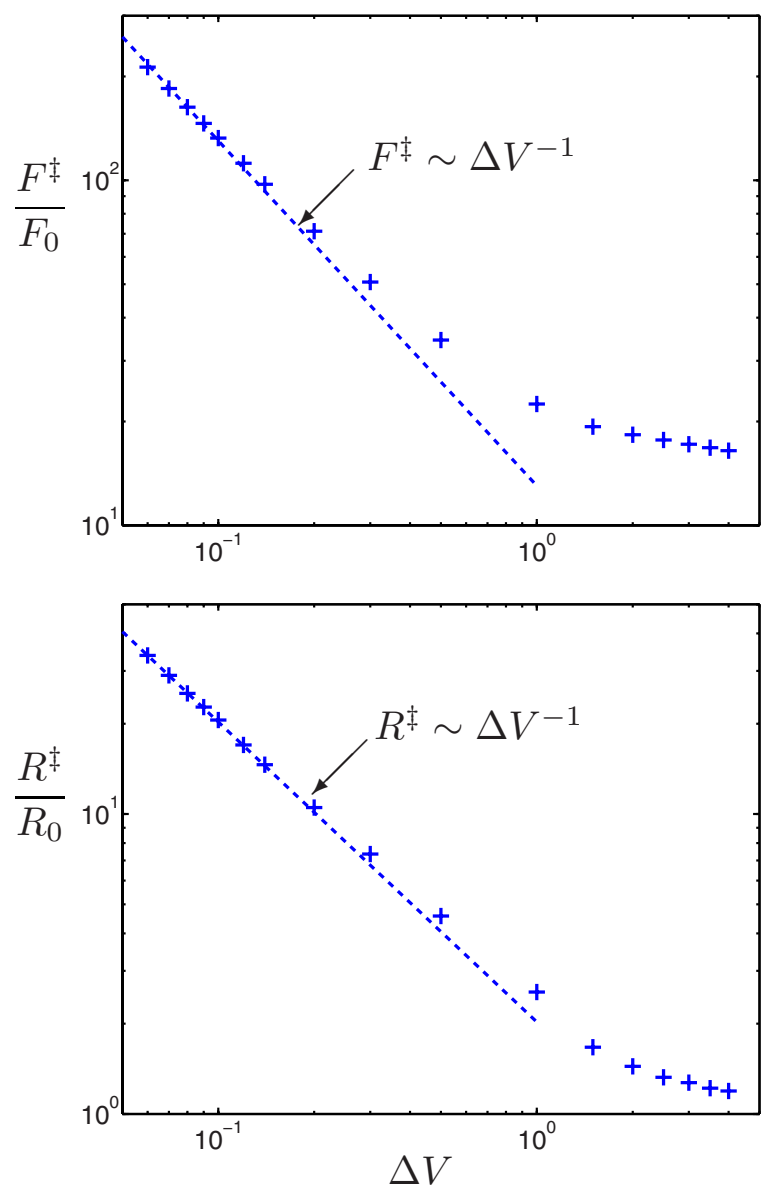

FIG. 7. (Color online) The saddle point free energy $F^{\ddagger}$ and the critical shape radius $R^{\ddagger}$ at different potential depths. The potential is identical to that in Fig. 6 with changing $\Delta V$. The radius $R$ is defined as the radial size of the contact area within the adhesion potential well: in our case the barrier is fixed at $z=0$; therefore, the radius is given by the size of the contact with $z \leq 0$.

analysis suggests that in the bending dominant regime the controlling length scale is $R_{0}=\left(\kappa L_{0}^{2} / V_{2}\right)^{1 / 4}$, which is independent of the potential depth difference, in particular the interfacial width $\Delta R \sim R_{0}$. In our calculations, the length scale of the adhesion potential $L_{0}$ is roughly kept constant; therefore, $R_{0}$ is constant and the geometry of membrane shapes is similar. On the other hand, the radius of the critical droplet increases as $\Delta V$ decreases and the energy barrier is determined by the potential depths. Therefore we conclude that the potential depths determine the energetics of nucleation, while the length scale $R_{0}$ controls the evolution of membrane shapes along the nucleation path.

To verify the dependence of nucleation on the energyminimum separation $\Delta L$, we calculate the energy barrier $F^{\ddagger}$ and the critical radius $R^{\ddagger}$ for the interaction potential $V(z)$ with potential minima having the same depths but varying locations. These results are shown in Fig. 8. The potential depths are fixed at $V_{1}=2$ and $V_{2}=1$, and the minima are located at $Z_{1}=-L$ and $Z_{2}=L$ with $L$ varying from 1 to 2 . We see that scaling relations $R^{\ddagger} \propto \Delta L^{1 / 2}$ and $F^{\star} \propto \Delta L$ fit well with numerical results.
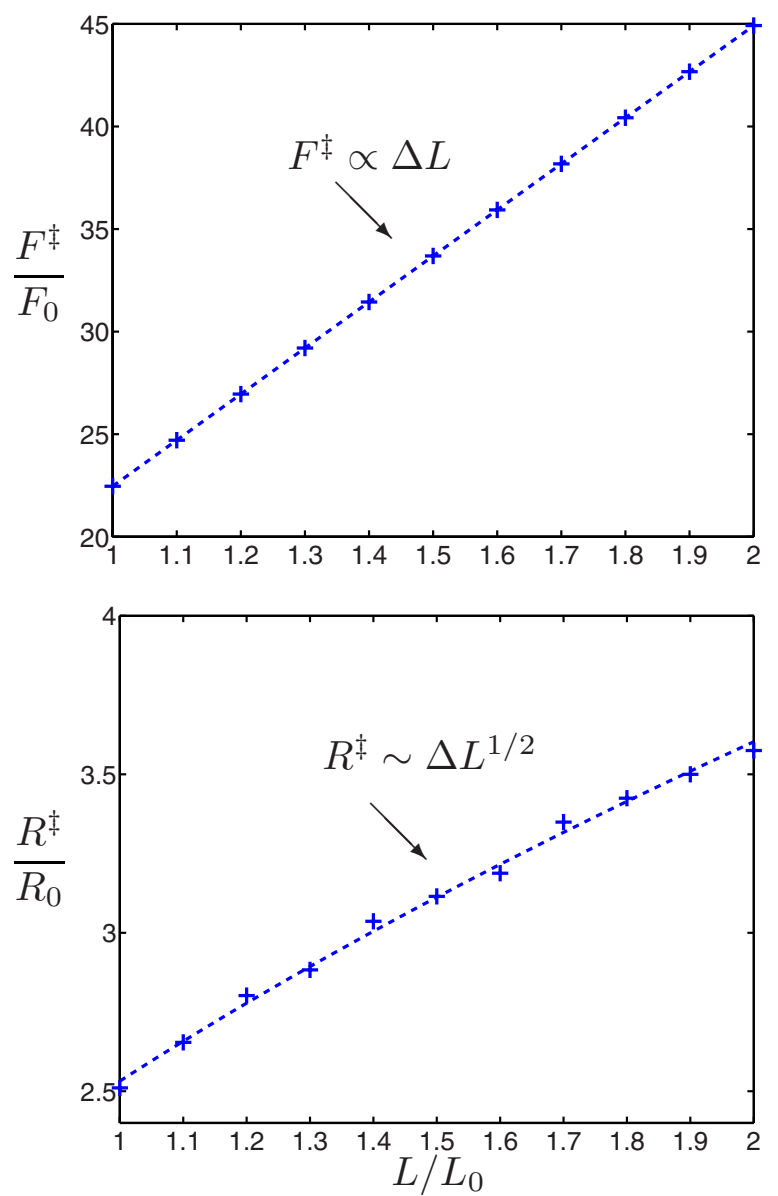

FIG. 8. (Color online) The saddle point free energy $F^{\ddagger}$ and the critical shape radius $R^{\ddagger}$ at different potential well separations. The potential wells are symmetrically positioned across the barrier with separation $\Delta L=2 L$ ranging from 2 to 4 . The potential depths are fixed at $V_{1}=2$ and $V_{2}=1$. Linear fits are done for $F^{\star}$ against $\Delta L$ and $\log R^{\ddagger}$ against $\ln \Delta L$ and shown as dashed lines.

Finally we study the effects of the surface tension term. Figure 9(a) shows the development of an adhesion nucleus under strong surface tension $\Sigma=3$. The potential depths are $V_{1}=2$ and $V_{2}=1$, and the minima are located at $Z_{1}=-1$ and $Z_{2}=1$. Compared to the case with no surface tension, we observe that the membrane shape is flatter and the extra surface energy increases the critical nucleus size. Figure 9(b) shows the crossover of the critical membrane shape from the rigidity-dominant regime to the tension-dominant regime; the straightening of the membrane shape due to surface tension is apparent. In Fig. 9(c) we plot the energy barrier against the surface tension. Under a small surface tension, the size of the critical nucleus does not change much and is still determined by the adhesion length $R_{0}$; hence, the total surface area of the adhesion droplet is almost constant and the free energy should be a linear function of the surface tension: this expectation is borne out by the numerical results.

In summary, we have shown that the energy barrier of adhesion is determined by $F_{0}=\sqrt{\kappa V_{2} \Delta L^{2}}$ and the evolution of the membrane shapes is controlled by the adhesion length $R_{0}=\left(\kappa \Delta L^{2} / V_{2}\right)^{1 / 4}$. Our numerical calculations yield that in 

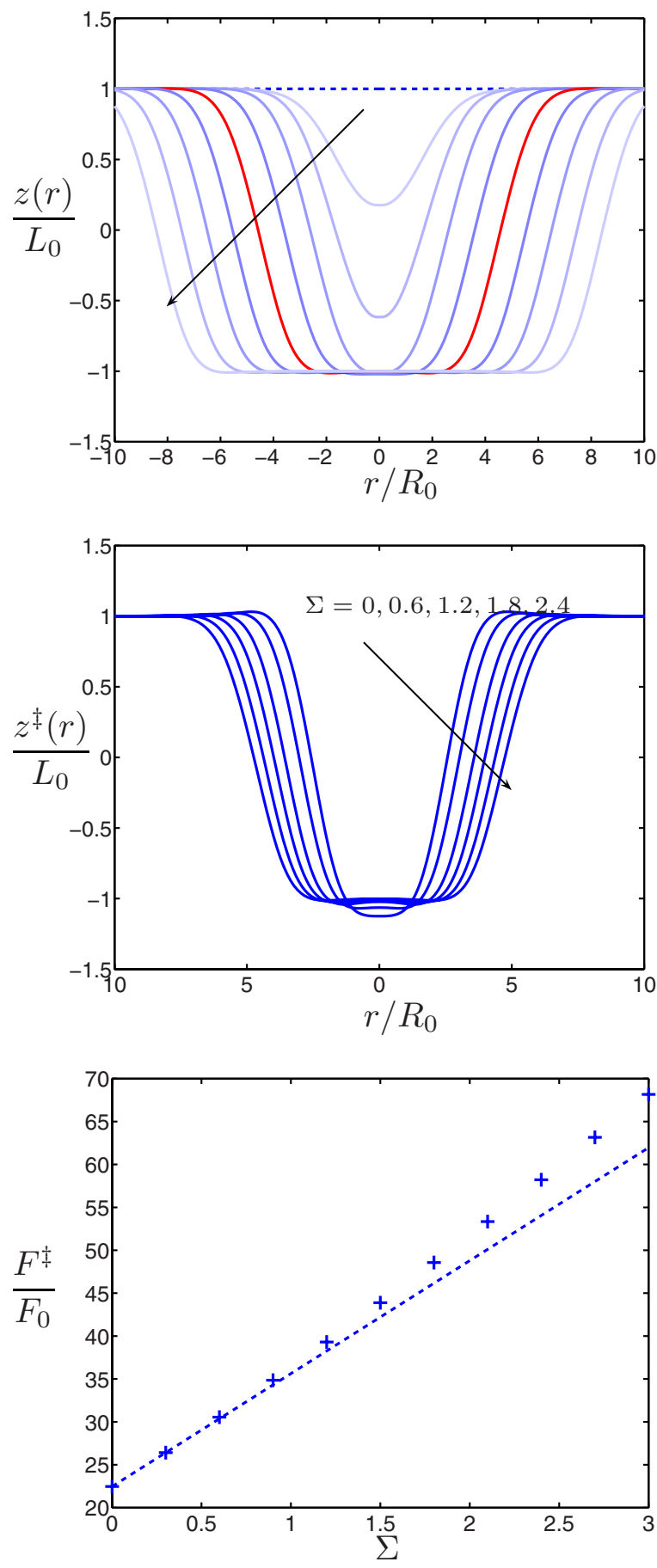

FIG. 9. (Color online) Illustration of the effects of surface tension. The potential is parametrized by $Z_{1}=-1, Z_{2}=1, V_{1}=2$, and $V_{2}=1$.

biomimetic membrane adhesion experiments, the adhesion barrier is about $20 k_{\mathrm{B}} T$ with a critical size of $50-100 \mathrm{~nm}$, while for cell adhesions nucleation may have a higher barrier and larger critical size, which is only to be overcome by active processes that may involve cell signaling. The scaling relations obtained from capillary approximations are in good agreement with numerical results. In addition, we find that the surface tension term flattens out the membrane shape and contributes an additional surface energy to the energy barrier that is a linear function of the surface tension when the tension is small.

A prominent example of cell adhesion controlled by a double-well potential is the formation of immunological synapses, which are focal contacts between a T-lymphocyte cell and an antigen-presenting cell (APC) [46]. The synapse primarily consists of the T-cell receptor (TCR)-major histocompatibility molecule-peptide complex (MHC) bonds $(15 \mathrm{~nm})$ and integrin (ICAM-1/LFA-1) bonds (40 nm). At high TCR expression, a fully developed synapse with both TCR-MHC bonds and integrin bonds is formed [46,47]. When TCR expression is low (e.g., in premature T-cells), the binary system of TCR and integrin binding should exhibit a double-well interaction potential separated by a large barrier [34]. In this regime, only small transient contacts are observed in computer simulations $[34,48,49]$. Different kinetic scenarios for the synapse formation have been identified by Weikl and Lipowsky [49] who suggested that a significant nucleation barrier may exist in this regime. Our calculation yields an explicit quantitative estimate for this barrier, which can indeed be considerable due to the large separation $(\sim 25 \mathrm{~nm})$ and membrane rigidity $\left(\sim 100 k_{\mathrm{B}} T\right)$. In this regard, our results complement the studies by Chakraborty et al. and Lipowksy et al., which are limited to no barrier or small barriers.

\section{CONCLUSION}

In this paper we have systematically studied the nucleation of membrane adhesions mediated by specific receptor binding. Scaling analysis suggests that the geometry of the membrane shape evolution is controlled by the adhesion length $R_{0}$ while the energetics is controlled by the energy scale $F_{0}=\sqrt{\kappa V_{2} L_{0}^{2}}$, where $L_{0}$ is the length scale of the adhesion potential, $V_{2}$ is the barrier height, and $\kappa$ is the bending rigidity. By applying a minimum-energy-path calculation we find that the typical energy barrier for biomimetic membrane adhesion is of the order of $(20-30) k_{\mathrm{B}} T$, which corresponds to a nucleation time scale of the order of seconds to an hour; the typical size of a nucleus is found to be $100 \mathrm{~nm}$ which is comparable to the estimation by Boulbitch et al. [15]. For cell adhesion, the energy barrier should be significant and cannot be overcome by membrane undulations due to thermal fluctuations. This suggests that active processes, such as actin reorganization or receptor aggregation, are necessary to overcome the energy barrier.

Surface tension increases the nucleation barrier as well as the size of the critical nucleus. But we find that at a small surface tension, the shape of the nucleus is still controlled by the adhesion length $R_{0}$ which is almost unaffected by the surface tension and the total surface area of the critical adhesion droplet is almost invariant. We also show that the nonmonotonic feature in the membrane shape near the phase boundary (as is first found in Ref. [45]) is due to the bending energy term and is reduced at increasing surface tension.

\section{ACKNOWLEDGMENT}

This work is supported by the National Science Foundation through MRSEC-Caltech. 
[1] B. Alberts, A. Johnson, J. Lewis, M. Raff, K. Roberts, and P. Walter, Molecular Biology of the Cell, 4th ed. (Garland, New York, 2002).

[2] J. M. Berg, J. L. Tymoczko, and L. Stryer, Biochemistry, 5th ed. (Freeman, New York, 2002).

[3] T. A. Springer, Nature (London) 346, 425 (1990).

[4] D. A. Lauffenburger and J. J. Linderman, Receptors: Models for Binding, Trafficking and Signaling (Oxford University Press, New York, 1993).

[5] R. Lipowsky, in Structure and Dynamics of Membranes Generic and Specific Interactions, edited by R. Lipowsky and E. Sackmann, Handbook of Biological Physics (Elsevier, Amsterdam, 1995), p. 521.

[6] Statistical Mechanics of Membranes and Surfaces, edited by D. Nelson, T. Piran, and S. Weinberg, 2nd ed. (World Scientific, Singapore, 2004).

[7] G. I. Bell, Science 200, 618 (1978).

[8] G. I. Bell, M. Dembo, and P. Bongrand, Biophys. J. 45, 1051 (1984).

[9] D. C. Torney, M. Dembo, and G. I. Bell, Biophys. J. 49, 501 (1986).

[10] D. Coombs, M. Dembo, C. Wolfsy, and B. Goldstein, Biophys. J. 86, 1408 (2004).

[11] Physics of Biological Systems: From Molecules to Species, edited by H. Flyvbjerg, J. Hertz, M. H. Jensen, O. G. Mouritsen, and K. Sneppen, Lecture Notes in Physics (Springer-Verlag, Berlin, 1997).

[12] D. Zuckerman and R. Bruinsma, Phys. Rev. Lett. 74, 3900 (1995).

[13] R. Lipowsky, Phys. Rev. Lett. 77, 1652 (1996).

[14] R. Bruinsma, A. Behrisch, and E. Sackmann, Phys. Rev. E 61, 4253 (2000).

[15] A. Boulbitch, Z. Guttenberg, and E. Sackmann, Biophys. J. 81, 2743 (2001).

[16] R. Bruinsma and E. Sackmann, in Physics of Biomolecules and Cells, Vol. 75 of Proceedings of the Les Houches Summer School of Theoretical Physics, edited by H. Flyvbyerg, P. Ormos, and F. David (Springer, Berlin, 2002), pp. 287-309.

[17] E. Sackmann and R. F. Bruinsma, ChemPhysChem 3, 262 (2002).

[18] E. Sackmann and S. Goennenwein, Prog. Theor. Phys. Suppl. 165, 78 (2006).

[19] T. A. Springer, Cell 76, 301 (1994).

[20] S. J. Singer and G. L. Nicolson, Science 175, 720 (1972).

[21] L. Peliti, in Fluctuating Geometries in Statistical Mechanics and Field Theory, Vol. 62 of Proceedings of the Les Houches Summer School of Theoretical Physics, edited by F. David, P. Ginzparg, and J. Zinn-Justin (Elsevier, Amsterdam, 1994), pp. 195-286.
[22] S. A. Safran, Statistical Thermodynamics of Surfaces, Interfaces and Membranes, Vol. 90 of Frontiers in Physics (Addison-Wesley, Reading, MA, 1994).

[23] R. Lipowsky, J. Phys. II 4, 1755 (1994).

[24] R. Lipowsky and S. Leibler, Phys. Rev. Lett. 56, 2541 (1986).

[25] L. Peliti and S. Leibler, Phys. Rev. Lett. 54, 1690 (1985).

[26] E. Sackmann, Science 271, 43 (1996).

[27] M. Tanaka and E. Sackmann, Nature (London) 437, 656 (2005).

[28] A. Albersdörfer, T. Feder, and E. Sackmann, Biophys. J. 73, 245 (1997).

[29] A. Kloboucek, A. Behrisch, J. Faix, and E. Sackmann, Biophys. J. 77, 2311 (1999).

[30] R. Simson, E. Wallraff, J. Faix, J. Nienöhner, G. Gerisch, and E. Sackmann, Biophys. J. 74, 514 (1998).

[31] J. Rädler and E. Sackmann, J. Phys. II 3, 727 (1993).

[32] J. O. Rädler, T. J. Feder, H. H. Strey, and E. Sackmann, Phys. Rev. E 51, 4526 (1995).

[33] A. Volmer, U. Seifert, and R. Lipowsky, Eur. Phys. J. B 5, 811 (1998).

[34] S. Raychaudhuri, A. K. Chakraborty, and M. Kardar, Phys. Rev. Lett. 91, 208101 (2003).

[35] W. E, W. Ren, and E. Vanden-Eijnden, Phys. Rev. B 66, 052301 (2002).

[36] M. Müller, K. Katsov, and M. Schick, Phys. Rep. 434, 113 (2006).

[37] W. Helfrich, Z. Naturforsch. C 28, 693 (1973).

[38] Z. Guttenberg, B. Lorz, E. Sackmann, and A. Boulbitch, Europhys. Lett. 54, 826 (2001).

[39] T. R. Weikl, D. Andelman, S. Komura, and R. Lipowsky, Eur. Phys. J. E 8, 59 (2002).

[40] T. R. Weikl, J. T. Groves, and R. Lipowsky, Europhys. Lett. 59, 916 (2002).

[41] H.-Y. Chen, Phys. Rev. E 67, 031919 (2003).

[42] E. Sackmann, J. Phys.: Condens. Matter 18, R785 (2006).

[43] M. Asfaw, B. Rózycki, R. Lipowsky, and T. R. Weikl, Europhys. Lett. 76, 703 (2006).

[44] J. I. Martin, C.-Z. Zhang, and Z.-G. Wang, J. Polym. Sci., Part B: Polym. Phys. 44, 2621 (2006).

[45] S. Komura and D. Andelman, Eur. Phys. J. E 3, 259 (2000).

[46] A. Grakoui, S. K. Bromley, C. Sumen, M. M. Davis, A. S. Shaw, P. M. Allen, and M. L. Dustin, Science 285, 221 (1999).

[47] S.-Y. Qi, J. T. Groves, and A. K. Chakraborty, Proc. Natl. Acad. Sci. U.S.A. 98, 6548 (2001).

[48] S.-J. E. Lee, Y. Hori, and A. K. Chakraborty, Proc. Natl. Acad. Sci. U.S.A. 100, 4383 (2003).

[49] T. R. Weikl and R. Lipowsky, Biophys. J. 87, 3665 (2004).

[50] C.-Z. Zhang and Z.-G. Wang, Langmuir 23, 13024 (2007).

[51] http://www.math.utah.edu/ eyre/research/methods/papers.html 